\title{
The association between the levels of autoimmune related indicators and clinical condition in sudden deafness patients
}

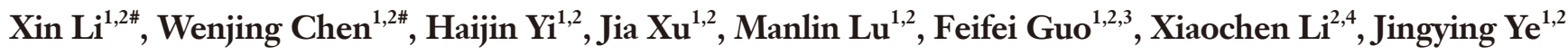 \\ ${ }^{1}$ Department of Otorhinolaryngology, Beijing Tsinghua Changgung Hospital, Beijing, China; ${ }^{2}$ School of Clinical Medicine, Tsinghua University, \\ Beijing, China; ${ }^{3}$ Department of Otorhinolaryngology, Xinjiang Bazhou People's Hospital, Xinjiang, China; ${ }^{4}$ Department of Laboratory, Beijing \\ Tsinghua Changgung Hospital, Beijing, China \\ Contributions: (I) Conception and design: X Li, W Chen; (II) Administrative support: X Li, J Ye; (III) Provision of study materials or patients, X Li, W \\ Chen, H Yi, J Xu, X Li; (IV) Collection and assembly of data: M Lu, F Guo; (V) Data analysis and interpretation: M Lu; (VI) Manuscript writing: All \\ authors; (VII) Final approval of manuscript: All authors. \\ \#These authors contributed equally to this work. \\ Correspondence to: Jingying Ye. Department of otorhinolaryngology, Beijing Tsinghua Changgung Hospital, 168 Litang Road, Changping district, \\ Beijing 102218, China. Email: yejingying@yeah.net.
}

\begin{abstract}
Background: This study aimed to analyze the abnormal rates of immune related factors in serum of sudden deafness (SD) patients, and to discuss the correlation between these indicators and the degree of pathological changes of SD patients.

Methods: From March 2018 to January 2021, 90 patients with SD who were hospitalized in Beijing Tsinghua Changgeng Hospital were enrolled as participants, and 60 healthy examinees served as the control group. Antinuclear antibody (ANA), anticardiolipin antibody immunoglobulin G (ACA-IgG), anticardiolipin antibody immunoglobulin M (ACA-IgM), and anti- $\beta$-glucoprotein (anti- $\beta$-GP) antibody were detected by enzyme-linked immunosorbent assay (ELISA).

Results: In the SD group, 43 participants were positive for ANA, while in the control group, only 9 participants were positive for ANA. The titer of antibodies was significantly higher in the SD group than in the control group. The differences in abnormal rates of ACA-IgG, ACA-IgM, and anti- $\beta-G P$ antibodies between the SD group and control group were not statistically significant $(\mathrm{P}>0.05)$.

Conclusions: The pathogenesis of SD is correlated with autoimmune factors to a certain extent. In the course of clinical treatment, in addition to vasodilation, neurotrophic therapy, and thrombolytic therapy, patients can also be approached from the perspective of autoimmunity, which may achieve more ideal treatment effects.
\end{abstract}

Keywords: Sudden deafness (SD); typing; antinuclear antibody; anticardiolipin antibody IgG; anticardiolipin antibody $\operatorname{Ig} M$

Submitted Jul 29, 2021. Accepted for publication Oct 16, 2021.

doi: 10.21037/apm-21-2388

View this article at: https://dx.doi.org/10.21037/apm-21-2388

\section{Introduction}

Sudden deafness (SD) is a common emergency in otolaryngology. Its incidence is approximately $[2.5-5] / 10,000$, the rate is similar between males and females, and the most common onset age is $40-60$ years old. In recent years, the incidence of SD has significantly increased $(1,2)$, although its etiology and pathogenesis are still unclear. A study revealed that a clear cause can be found in only approximately $10 \%$ of SD patients, and the majority of cases are largely idiopathic (3). The entity was first described by De Kleyn in 1944. The etiology and pathogenesis of sudden deafness remain unknown. Proposed theories of causation include vascular occlusion, viral infection, breaks of labyrinthine membranes, immune- 
mediated mechanisms and abnormal cellular stress responses within the cochlea (4).

At present, the pathogenesis of SD is mainly attributed to microcirculatory disorder of the inner ear, viral infection, and rupture of the labyrinth membrane. Sensorineural hearing loss is characterized by degeneration of key structures of the sensory pathway in the cochlea, such as the sensory hair cells, the primary auditory neurons and their synaptic connection to the hair cells - the ribbon synapse (5). Li et al. (6) investigated the relationship between $\mathrm{T}$ cell subsets in peripheral blood and the incidence and prognosis of sudden deafness. Compared with the control group, $\mathrm{CD}^{+}, \mathrm{CD}^{+}$and $\mathrm{CD} 4^{+} / \mathrm{CD}^{+}$in peripheral blood of the study group were significantly lower before treatment, while $\mathrm{CD}^{+}$was significantly higher, the difference was statistically significant $(\mathrm{P}<0.05)$. They make a conclusion that there is a relationship between the occurrence of sudden deafness and the imbalance of $T$ cell subsets in peripheral blood. Immune dysfunction may affect the hearing recovery of patients with sudden deafness.

Autoimmune factors have been considered one of the important causes of SD $(7,8)$. A clinical trial report revealed that the remission rate of steroid treatment for SDs was higher than $70 \%$, and the response rate of placebo treatment was only $38 \%$, which confirms the role of the immune system in the pathogenesis of SD. Studies from outside of China have confirmed that in approximately $1 / 3$ of patients, SD was secondary to systemic autoimmune diseases $(9,10)$. Autoimmune diseases are usually systemic diseases, and the pathological process is complicated. Most of the antibodies tested currently have no obvious specificity for the inner ear, therefore, the correlation between the occurrence of SD and autoimmune factors still requires exploration through further laboratory and clinical studies.

In the present study, we analyzed the abnormal rate of antinuclear antibodies (ANA) and that of expression levels of anti-cardiolipin antibodies (ACA) such as immunoglobulin G (ACA-IgG), immunoglobulin M (ACA-IgM), and anti- $\beta$ glycoprotein (anti- $\beta-\mathrm{GP}$ ) antibodies in the serum of patients with $\mathrm{SD}$, to preliminarily investigate the correlation between the above indicators and the degree of disease in patients with SD. In doing so, we hoped to find objective indicators that can help reflect the pathological changes of patients with SD, in order to provide a theoretical reference for clinical diagnosis, disease assessment, and efficacy judgment. We present the following article in accordance with the STROBE reporting checklist (available at https:// dx.doi.org/10.21037/apm-21-2388).

\section{Methods}

\section{Participants}

From March 2018 to January 2021, 90 patients with SD who were hospitalized at the Beijing Tsinghua Changgung Hospital were enrolled as study participants, and 60 healthy individuals served as the control group. The inclusion criteria stipulated that the patients met the diagnostic criteria for SD, as developed by the Chinese Medical Association Otolaryngology Head and Neck Surgery Branch (11). Its criteria are as follows: (I) sudden occurrence, the disease occurs within a few minutes, hours, or over 3 days; (II) nonfluctuating sensorineural hearing loss, mild, moderate, or severe, even total deafness; hearing diminished by more than $20 \mathrm{~dB}$ at least at 2 connected frequencies; mostly occurs in unilateral ear, occasionally occurs in bilateral ears simultaneously or successively; (III) pathogeny is unknown (no clear cause is found including systemic or local factors); (IV) accompanied by tinnitus, and a sensation of aural stuffiness; (V) may be accompanied by dizziness, nausea, or vomiting, but not repeated attacks; (VI) except for the eighth cranial nerve, there are no symptoms of damage to other cranial nerves.

\section{Informed consent}

The research plan was approved by the Medical Ethics Committee of Beijing Tsinghua Changgung Hospital before implementation (20150911-05). The researcher clearly delineated the research objectives, specific implementation steps, and possible challenges to the participants. All patients and healthy controls enrolled in the present study clearly understood the purpose and specific implementation plan of the study, and provided written informed consent. The study was conducted in accordance with the Declaration of Helsinki (as revised in 2013).

\section{Blood specimen collection}

On the second day after admission, $5 \mathrm{~mL}$ of fasting peripheral blood was collected from each participant in the experimental and control groups in the morning. Anticoagulation was not performed, the blood was centrifuged at 3,000 rpm for $10 \mathrm{~min}$, and then the serum was obtained.

\section{Detection of ANA, ACA-IgG, ACA-IgM, and anti- $\beta-G P$ antibody by enzyme-linked immunosorbent assay}

All operations were conducted according to the enzyme- 
Table 1 Comparison of abnormal rates of ANA in different types of SD patients

\begin{tabular}{|c|c|c|c|c|c|c|}
\hline Curve type & ANA positive (n) & ANA negative $(n)$ & Total (n) & Positive rate (\%) & $\chi^{2}$ & $P$ value \\
\hline High frequency type & 5 & 8 & 13 & 38.46 & 25.52 & $<0.01$ \\
\hline Planar type & 10 & 12 & 22 & 45.45 & 3.29 & $>0.05$ \\
\hline Total deafness & 11 & 14 & 25 & 44.00 & 2.98 & $>0.05$ \\
\hline
\end{tabular}

ANA, anti-nuclear antibodies; SD, sudden deafness.

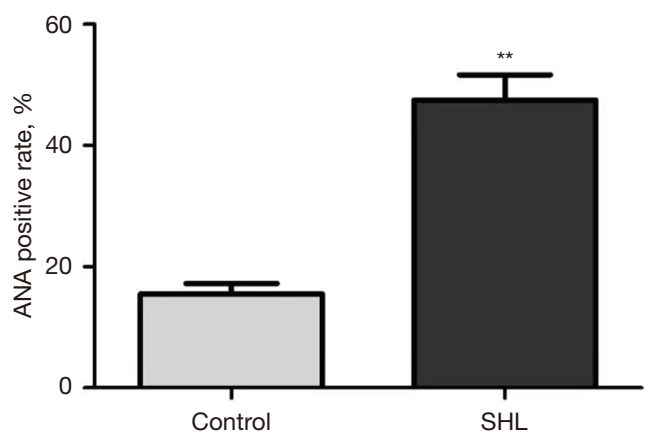

Figure 1 Comparison of abnormal rates of ANA in different types of SD patients. ${ }^{* *}, \mathrm{P}<0.01$. ANA, anti-nuclear antibodies; SD, sudden deafness.

linked immunosorbent assay (ELISA) kit instructions. Detection of ANA was conducted using indirect immunofluorescence. The detection of the ANA series was conducted using Euromont blotting. The normal threshold of ACA-IgG was set at $12 \mathrm{GPL}$ unit/mL, a result with a value higher than this critical value was defined as positive, and that with a value lower than this critical value was defined as negative. The normal threshold of ACA-IgM was set at $12 \mathrm{MPL}$ unit $/ \mathrm{mL}$, a result with a value higher than this critical value was defined as positive, and that with a value lower than the critical value was defined as negative. The normal threshold of anti- $\beta$-GP antibody was set at $20 \mathrm{RU}$ unit $/ \mathrm{mL}$, the result with a value higher than this critical value was defined as positive, and that with a value lower than this critical value was defined as negative. All kits were produced by Euroimmun (Lübeck, Germany).

\section{Statistical analysis}

All data in the present study were statistically analyzed using the software SPSS 19.0 (IBM Corp., Chicago, IL, USA). All measurement data were tested for normality (K-S test). Normally distributed data were expressed as the mean \pm standard deviation (SD), and compared between groups using univariate analysis of variance. Inspection level was $\alpha=0.05$. Statistical significance was considered at $\mathrm{P}<0.05$.

\section{Results}

\section{Comparison of general situations between $S D$ patients and bealthy controls}

A total of 90 patients with SD were enrolled. The age of these patients was $27-83$ years, and the median age was 54.91 years. Among these patients, 38 were male, and 52 were female. The course of disease ranged between 2 days and 18 months. They were classified according to hearing curve: 25 participants had total deafness, 13 had high frequency type deafness, 30 had low frequency type deafness, and 22 had planar type deafness. Among this patient group, 45 participants had tinnitus, and 38 had dizziness. A total of 60 healthy individuals were assigned to the control group. The age of these participants was 18 70 years, and the median age was 52.58 years (Table 1).

In the SD group, ANA were detected as positive in 43 participants, while in the control group, ANA were detected as positive in only 9 participants. Furthermore, the antibody titer was significantly higher in participants in the SD group than in the control group $(\mathrm{P}<0.05$, Figure 1).

The differences in abnormal rates of ACA-IgG, ACAIgM, and anti- $\beta-G P$ antibody between the SD group and control group were not statistically significant $(\mathrm{P}>0.05)$.

\section{Discussion}

SD refers to a sharp decline in hearing that occurs in a short period of time (or more than 48 hours), without an obvious trigger. The disease usually occurs on one side, and rarely occurs on both sides. Pure tone hearing test result manifests as a hearing loss of $>20 \mathrm{~dB}$ at least at 3 connected frequencies, and those affected often experience tinnitus, 
dizziness, nausea, vomiting and other symptoms, but have no recurrent attacks, and except for the $8^{\text {th }}$ pair of cranial nerves, no other cranial nerves are damaged (12). Due to its swift onset and rapid development of the condition, it is one of common emergencies in otolaryngology. If the patient is not treated with effective comprehensive treatment, it becomes difficult to recover hearing on the affected side, and it may develop into a permanent neurological deafness, seriously affecting the quality of life of the patient.

The reason why leukocytes increase in patients with sudden deafness is that leukocytes, especially neutrophils, are an important part of the body's defense system, known as "body guard", and pathological neutrophils increase can be seen in: acute infection: bacteria, certain virus is like epidemic bleach blood heat, epidemic encephalitis of type $\mathrm{B}$, rabies, increase the degree and the kind of pathogen, infection place and degree and airframe reaction sex concern, be like the mild infection of focal sex, total number of leucocyte can be in normal range, neutrophil percentage increases only. tissue damage or necrosis severe trauma, surgical trauma, vascular embolism, etc. Leukocytes often increase in acute poisoning, mainly neutrophils. Others such as corticosteroids. Therefore, the mechanism of leukocyte increase in peripheral blood of patients with sudden deafness may be a manifestation of stress response and tissue injury stimulation, or there are some infectious factors. And the increase of white blood cells in the surrounding blood has a bad effect on the prognosis of sudden deafness (13). The incidence of SD is increasing annually, and its age of onset is decreasing. At present, the cause and pathogenesis of the disease are still unclear; however, it may be that the structure and function of the inner ear are altered by ischemia and hypoxic damage of hair cells, caused by various factors. In recent years, Chinese and international researchers have tried to explore the role of autoimmune factors in the pathogenesis of SD $(11,14)$. The present study aimed to explore the clinical significance of autoimmune indicators in patients with SD by detecting changes in the levels of ACA-IgG, ACA-IgM, anti-B2- $\beta$ GP, and ANA in plasma of patients with SD. The present study revealed that the difference in ANA between patients with SD and healthy controls was statistically significant. However, in the present study, the differences in ACA-IgG, ACA-IgM, and $\beta-G P$ antibodies between patients with SD and healthy controls were not statistically significant.

The term ANA now broadly refers to an umbrella of autoantibodies to all antigenic components in cells. At present, autoantibodies are mainly used for the detection of autoimmune system diseases. An increasing number of studies have revealed that autoimmune phenomena may be present in patients with SD (15). In the present study, the age of these patients was $27-83$ years old; and the age of healthy blood donors was $18-70$ years old. The positive rate of ANA was $47.78 \%$ in participants with SD, which was significantly higher than that in the healthy control group (15.58\%). This indicates that the probability of SD caused by autoimmune factors can be inferred by detecting changes in the level of ANA in serum, and drug intervention can be designed from this perspective.

Etiology and pathogenesis of sudden deafness are still controversial, but most scholars believe that microcirculation disorders caused by nerve, blood flow and metabolism disorders of the inner ear is one of the main etiology. Microcirculation disorder will affect the function of coagulation and fibrinolysis system to some extent, and change the related indexes of coagulation, anticoagulation and fibrinolysis system, so that blood is in a hypercoagulable state.

Antiphospholipid antibodies (aPL) are a class of heterogeneous antibodies which are associated with hypercoagulability. They are a group of polyclonal immunoglobulins present in circulating serum, and are one of the means of directing communication between the 2 major physiological systems of immunity and coagulation. The APLs are divided into 2 types: lupus anticoagulant (LA) and anti-cardiolipin antibody (ACL). The LAs are classified as $\operatorname{lgG}, \lg M$, or $\lg G / \operatorname{IgM}$ and bind to phospholipids in cell membranes (16-18). Some LAs have also anticardiolipin activity, which are identified as anti- $\beta 2$ glycoprotein ( $\beta 2-G P 1)$ autoantibodies. The ACLs are divided into 3 categories: IgG, LeA, and $\operatorname{LgM}$, which can bind to cardiolipin, phosphatidylserine, and phosphalidylinosilol. Toubi et al. (19) studied 30 patients, among whom 11 had SD, and 19 had progressive sensorineural deafness. Low-to-moderate titers of anticardiolipin antibodies were found in $8(27 \%)$ of all 30 participants $(\mathrm{P}<0.02)$, and 5 of these 8 participants had SD. Studies revealed that, hypertension was a common symptom in patients with high ACL antibody titers, and one of its important manifestations was the presence of vasculopathy. Among the causes of SD, inner ear microcirculatory disorder is currently considered one of the most important $(19,20)$. In addition, some stimulating factors, such as microorganisms, radiation, and chemicals, can produce an immune response in normal inner ear components. A related study revealed that activated $\mathrm{T}$ lymphocytes and adhesion molecules can 
penetrate the inner ear through the capillary wall of the brain, causing a series of immune effects (21). Treg cells are considered to be involved in the pathogenesis of inner ear disease, its pathological mechanism is mainly that activated $\mathrm{T}$ lymphocytes pass through the blood-brain barrier under the influence of various factors, which can enter the brain parenchyma, after re-stimulation of a series of local antigens in the brain parenchyma, these $T$ cells can accelerate the synthesis in brain parenchyma, then a large number of chemokines and cytokines are released. These chemokines and cytokines can recruit $\mathrm{T}$ lymphocytes and $\mathrm{B}$ cells in the peripheral circulation to enter the inner ear, and the above cells entering the inner ear can gradually enlarge the immune response, causing damage to the inner ear $(22,23)$. Therefore, glucocorticoid therapy to improve the edema state of microvascular endothelial cells in the inner ear, increase the blood flow of small blood vessels in the inner ear, and improve the ischemia and hypoxia in the inner ear, is still the main treatment for SD. However, in the present study, in 90 participants with SD, ACA-IgM were detected as positive in only 6 participants, and the difference was not statistically significant when compared with the control group. No SD patients were detected to be positive for ACA-IgG and anti- $\beta-G P$ antibody.

Autoimmune inner ear disease is considered to be an important pathogenic factor of sudden deafness. Immune factors can be primary reactions of the inner ear, or secondary to systemic autoimmune diseases such as Wegener's granuloma, systemic lupus erythematosus, rheumatoid arthritis, polyarteritis nodosa, Cogan syndrome, etc. Studies have shown (24) that the inner ear labyrinth has the ability of rabbit epidemic response, immune defense and immune regulation. In some pathological conditions, the inner ear tissue can become its own antigen and stimulate its own rabbit epidemic response, resulting in the destruction of inner ear tissue and function, and then the hearing loss. It has been reported that a variety of immune markers have been found in the serum of patients with sudden deafness. For example, some studies suggest that the autoimmunity of collagen tissue may be one of the causes of the disease, and some authors believe that immune-induced vascular injury plays an important role in the pathogenesis of sudden deafness. Although the above studies suggest the relationship between sudden deafness and autoimmune inner ear diseases, further research is needed due to the extremely complex pathogenesis and pathogenesis of autoimmune diseases.

The occurrence of sudden deafness is related to many factors, among which microcirculation disorder, immune damage, virus infection and endolymphatic hydrocephalus have been gradually recognized as the main pathogenesis. Stress theory is an important pathogenesis theory of sudden deafness proposed in recent years, which can macroscopically explain the pathophysiological process of sudden deafness from many angles. It is not only one of the causes of sudden deafness, but also a secondary factor of other causes. As for the treatment of sudden deafness, the Guidelines of China (25), the Guidelines of the United States (26) and The Guidelines of Germany (27) all recommend systemic administration of glucocorticoids in the acute stage of sudden deafness, and drum injection of glucocorticoids as remedial treatment when the systemic administration effect is not good. The consensus of experts on hyperbaric oxygen therapy for sudden deafness in China in 2018 points out that patients with sudden deafness should receive hyperbaric oxygen therapy as soon as possible. Hyperbaric oxygen therapy is effective within 3 months of onset, and the time of hyperbaric oxygen remedial therapy can be extended to 6 months. In addition, there is no special description of the guidelines for improving circulation, the use of nutritional nerve drugs, and the treatment of Traditional Chinese medicine at home and abroad, and the pathophysiological mechanism of action and curative effect are certain, so in the actual clinical work can use a variety of methods for the treatment of sudden deafness.

\section{Conclusions}

In summary, the pathogenesis of SD is correlated with autoimmune factors to a certain extent. Therefore, in the clinical therapeutic process, in addition to vasodilation, neurotrophic therapy, and thrombolytic therapy, patients can also be treated from the perspective of autoimmunity, in order to optimize treatment effects.

\section{Acknowledgments}

Funding: National Natural Science Foundation of China (81670918, 81873696), Beijing Municipal Administration of Hospitals Incubating Program (PX2016031).

\section{Footnote}

Reporting Checklist: The authors have completed the STROBE reporting checklist. Available at https://dx.doi. org/10.21037/apm-21-2388 
Data Sharing Statement: Available at https://dx.doi. org/10.21037/apm-21-2388

Conflicts of Interest: All authors have completed the ICMJE uniform disclosure form (available at https://dx.doi. org/10.21037/apm-21-2388). The authors have no conflicts of interest to declare.

Ethical Statement: The authors are accountable for all aspects of the work in ensuring that questions related to the accuracy or integrity of any part of the work are appropriately investigated and resolved. The study was conducted in accordance with the Declaration of Helsinki (as revised in 2013). The study was approved by the Medical Ethics Committee of Beijing Tsinghua Changgung Hospital (20150911-05). All patients and healthy controls enrolled in the present study clearly understood the purpose and specific implementation plan of the study, and provided written informed consent.

Open Access Statement: This is an Open Access article distributed in accordance with the Creative Commons Attribution-NonCommercial-NoDerivs 4.0 International License (CC BY-NC-ND 4.0), which permits the noncommercial replication and distribution of the article with the strict proviso that no changes or edits are made and the original work is properly cited (including links to both the formal publication through the relevant DOI and the license). See: https://creativecommons.org/licenses/by-nc-nd/4.0/.

\section{References}

1. Editorial Board of Chinese Journal of Otolaryngology Head and Neck Surgery, Society of Otolaryngology Head and Neck Surgery, Chinese Medical Association. Guidelines for diagnosis and treatment of sudden deafness (2015). Chinese Journal of Otolaryngology Head and Neck Surgery 2015;50:443.

2. Shi X. Physiopathology of the cochlear microcirculation. Hear Res 2011;282:10-24.

3. Lobo DR, García-Berrocal JR, Ramírez-Camacho R. New prospects in the diagnosis and treatment of immune-mediated inner ear disease. World J Methodol 2014;4:91-8.

4. Merchant SN, Durand ML, Adams JC. Sudden deafness: is it viral? ORL J Otorhinolaryngol Relat Spec 2008;70:5260; discussion 60-2.

5. Ma Y, Wise AK, Shepherd RK et al. New molecular therapies for the treatment of hearing loss. Pharmacol Ther 2019;200:190-209.

6. Li W, She W, Wu X, et al. Relationship between T cell subsets of peripheral blood and sudden deafness. China Medical Guide 2019;16:106-8.

7. Bundhun PK, Soogund MZS, Huang F. Arterial/venous thrombosis, fetal loss and stillbirth in pregnant women with systemic lupus erythematosus versus primary and secondary antiphospholipid syndrome: a systematic review and meta-analysis. BMC Pregnancy Childbirth 2018;18:212.

8. Facciabene A, Motz GT, Coukos G. T-regulatory cells: key players in tumor immune escape and angiogenesis. Cancer Res 2012;72:2162-71.

9. Li R, Yang W, Zhang J, et al. Association of CD247 with systemic lupus erythematosus in Asian populations. Lupus 2012;21:75-83.

10. Cadoni G, Agostino S, Manna R, et al. Clinical associations of serum antiendothelial cell antibodies in patients with sudden sensorineural hearing loss. Laryngoscope 2003;113:797-801.

11. Furuhashi A, Matsuda K, Asahi K, et al. Sudden deafness: long-term follow-up and recurrence. Clin Otolaryngol Allied Sci 2002;27:458-63.

12. Masindova I, Varga L, Stanik J, et al. Molecular and hereditary mechanisms of sensorineural hearing loss with focus on selected endocrinopathies. Endocr Regul 2012;46:167-86.

13. Zhang M, Zhong GY. Correlation between prognosis and increase of leucocyte count for the sudden hearing loss. Journal of Otolaryngology and Ophthalmology of Shandong University 2006;20:111-3.

14. Liu Y, Jiang W, Mao K, et al. The evaluating time of curative effect on sudden deafness. Lin Chung Er Bi Yan Hou Tou Jing Wai Ke Za Zhi 2012;26:308-11.

15. Tao D, Chen QE, Tan JL. The relationship of mental health and coping styles in patients with sudden deafness. Chinese Journal of Nursing 2012;47:150-1.

16. Park JJ, Jeong SW, Lee JW, et al. A Case of Sudden Deafness with Intralabyrinthine Hemorrhage Intralabyrinthine Hemorrhage and Sudden Deafness. J Audiol Otol 2015;19:178-81.

17. Lee EJ, Yoon YJ. Sudden deafness as an initial presenting symptom of posterior inferior cerebellar artery infarction: two case reports. J Laryngol Otol 2014;128:1011-4.

18. Zhang X, Zhou J, Tan G, et al. Clinical analysis of short-term efficacy in senile sudden deafness. Journal of Clinical Otorhinolaryngology Head and Neck Surgery 
2013;27:1272-3, 1275 .

19. Toubi E, Ben-David J, Kessel A, et al. Autoimmune aberration in sudden sensorineural hearing loss: association with anti-cardiolipin antibodies. Lupus 1997;6:540-2.

20. Zheng Z, Sun YY, Xia L, et al. Research progress on the relationship between sudden deafness and blood lipid. Journal of Shanghai Jiaotong University (Medical Edition) 2017;37:859-64.

21. Zhou XY, Bai Z. Research progress on the relationship between sudden deafness and microcirculatory disturbance of inner ear. Clinical Medicine 2015;35:122-4.

22. Du YS, Zhao LP, Yang J, et al. Anti-inner ear antibody and sudden deafness. Chinese Archives of OtolaryngologyHead and Neck Surgery 2011;7:81-2.

23. Jia X, Xia Y. Antiphospholipid antibody syndrome with central nervous system diseases: report of 5 cases and literature review. Nerve injury and functional reconstruction. 2016:525.

Cite this article as: $\mathrm{Li} \mathrm{X}$, Chen $\mathrm{W}, \mathrm{Yi} \mathrm{H}, \mathrm{Xu} \mathrm{J}, \mathrm{Lu}$ M, Guo F, $\mathrm{Li}$ X, Ye J. The association between the levels of autoimmune related indicators and clinical condition in sudden deafness patients. Ann Palliat Med 2021;10(10):10535-10541. doi: 10.21037/ apm-21-2388
24. Rossi O, Citiulo F, Mancini F. Outer membrane vesicles: moving within the intricate labyrinth of assays that can predict risks of reactogenicity in humans. Hum Vaccin Immunother 2021;17:601-13.

25. Yu L, Yang S. Guidelines for the diagnosis and treatment of sudden deafness (2015). Chinese Journal of Otorhinolaryngology Head and Neck Surgery 2015;50:443-7.

26. Chandrasekhar SS, Tsai Do BS, Schwartz SR, et al. Clinical Practice Guideline: Sudden Hearing Loss (Update). Otolaryngol Head Neck Surg 2019;161:S1-45.

27. Michel O; Deutsche Gesellschaft für Hals-Nasen-OhrenHeilkunde, Kopf- und Hals-Chirurgie. The revised version of the german guidelines "sudden idiopathic sensorineural hearing loss". Laryngorhinootologie 2011;90:290-3.

(English Language Editor: J. Jones) 